\title{
An efficient and optimized tracking framework through optimizing algorithm in a deep forest using NFC
}

\author{
Md. Abbas Ali Khan', Mohammad Hanif Ali ${ }^{2}$, A.K.M. Fazlul Haque ${ }^{3}$, Chandan Debnath ${ }^{4}$, \\ Shohag Kumar Bhowmik ${ }^{5}$ \\ 1,2 Department of Computer Science and Engineering, Jahangirnagar University, Bangladesh \\ ${ }^{1,5}$ Department of Computer Science and Engineering, Daffodil International University, Bangladesh \\ ${ }^{3}$ Department of ETE, Daffodil International University, Bangladesh \\ ${ }^{4}$ Department of Computer Science and Engineering, National University, Bangladesh
}

\section{Article Info \\ Article history: \\ Keywords: \\ Forest \\ NFC \\ NFC device \\ NFC tag \\ Tracking}

Received Dec 10, 2019

Revised Feb 11, 2020

Accepted Feb 25, 2020

\begin{abstract}
NFC is applying in various field of contemporary technology. Especially of convenience tag usability in any place. One of the facilities which can be added in the tracking system is the implementation of Near Field Communication in order to guide each tourist in the deep forest or any other location. In the deep forest, tracking or location detection activities need to be done efficiently, like desired path finding in a deep forest. At present, the tracking procedure in deep forest is working with the help of guides or local citizens. Currently, in any restricted area such as the "Sundarban" forest, no outside general people are allowed to travel in the jungle without any authorized guide which is not an efficient way to travel smoothly. The use of Near Field Communication can solve the problem related to lost the way, safety, and easily help the travelers to track the desired destination without the help of human resources or any guide. The NFC tags that hold mapping information of the area, in the point of tag setup all tags will be set up on several trees along with sequence.
\end{abstract}

Copyright $\odot 2020$ Institute of Advanced Engineering and Science. All rights reserved.

Corresponding Author:

Md. Abbas Ali Khan,

Department of CSE, Daffodil International University,

Ph.D. Scholar, Jahangirnagar University Bangladesh.

Email: abbas.cse@diu.edu.bd

\section{INTRODUCTION}

NFC (Near Field Communication) ISO/IEC 14443 and ISO/IEC 18000-3 devours the most reduced power than different wireless application protocol. It was begun in the right on time of the 1980s yet the structure in that time was RFID (Radio Frequency Identification). Charles Walton got the principal credit for an item utilizing the RFID innovation in 1983 [1]. This mechanization enabled the client to send radio data to a distinguished beneficiary. It is a magnificent innovation to utilize security labels in shrewd shops, bistro or any retail store. Specifically, NFC is a piece of High-Frequency (HF) RFID, and both work at the 13.56 MHz frequency [1]. NFC is planned to be an ensured sort of data exchange, and a NFC device is fit for being both a NFC per client and a NFC tag [1]. Coverage area of communication is $4-10 \mathrm{~cm} \mathrm{[2].}$

Many of the researcher they did in various field of research using NFC like, payment method system, payment security, airport baggage claim [3-5] and so on. In this paper it is proposed a path detection framework in a deep forest. So far it is known that there is no previous research has not done yet. This present study will discuss about the simulation of NFC protocol path tracking and data read features. By using the framework any traveler or new comer can track the correct path without help of any guide or local people if he or she is lost in the jungle and it will also a power consuming framework. At present this research is only conducted for deep forest but it also can be implemented in any tourist place or any other places to detect the desired location. 
NFC technology contains two terminology, one is NFC tag and another one is NFC device [6]. NFC tag is a used to store and transfer data to NFC device and also powering wireless sensor [7]. It doesn't require any power and it is cheaper than another device. The ratio of data loss is very low even though no data is lost and the data can be stored only for one time. So it shows that the written data are nonvolatile. In contrast NFC device is an electronic component which is used to read data from NFC tag. IT requires daily charging. Such as most of the android phones are NFC enable.

Another reason to choice this technology rather than another short range wireless protocol because NFC tag can be used without any charging system where as another short range wireless protocols requires charging continuously, hourly, daily or yearly [8]. So the use of NFC tag in deep forest is a perfect decision for the proposed method. Comparing to another short range wireless technologies NFC provides more power consuming facilities. More over it is stable than any other system in the open deep forest.

\section{PRACTICAL APPROACH}

The popularity of traveling is increasing gradually as infrastructures have been repaired and other supporting facilities have been added all over the world. At present, a problem is occurred often that the tracking procedure in deep forest is working with the help of guides or local citizens. Currently, in any restricted area such as the "Sundarban" forest, no outside general people are allowed to travel in the jungle without any authorized guide which is not an efficient way to travel smoothly. One of the facilities which can be added in the tracking system is the implementation of Near Field Communication in order to guide each tourist in the deep forest or any other location. In the deep forest, tracking or location detection activities need to be done efficiently, like desired path finding in a deep forest. The use of Near Field Communication can solve the problem related to lost the way, safety, and easily help the travelers to track the desired destination without the help of human resources or any guide [9].

NFC is an open platform, so that the standardization in using NFC in deep forest or tourist place is needed. The standardization which is necessary to be implemented is the one towards the speed transfer, protocol systems, and types of data, data string, authentication method, and validation method. The NFC has several operation modes including Peer-to-Peer (P2P), Reader/Writer (R/W), and NFC Card Emulation (NCE). These modes can be used for Mobile Transaction (Contactless Payment) [10-13], Data Exchange, and Read-Write.

\section{SYSTEM DESCRIPTION}

Now-a-days traveling is a popular event among all aged people. To make the travelling happy and secure the aforementioned framework is proposed a risk free path tracking system in the deep forest. When Figure 1 is referred that describe the working procedure of the proposed study method. All the mapping information will be visualized in the NFC device whenever it is touched on the NFC tag. A* search algorithm is consider for developing mapping information system [14]. It is an informed search algorithm, or a best-first search, implying that it is planned as far as weighted charts: beginning from a particular beginning node of a diagram, it intends to discover a way to the given desired node having the smallest cost (least distance traveled, shortest time, and so forth) [15]. The main formula of this search is given bellow which selects the minimum path

$$
\mathrm{f}(\mathrm{n})=\mathrm{g}(\mathrm{n})+\mathrm{h}(\mathrm{n})
$$

where $\mathrm{n}$ is the subsequent node on the way, $\mathrm{g}(\mathrm{n})$ is the cost of the path from the begin node to $\mathrm{n}$, and $\mathrm{h}(\mathrm{n})$ is a heuristic function that gauges the expense of the least expensive path from $n$ to the goal. $\mathrm{A}^{*}$ ends when the path it expands is a path from beginning to the goal or if there is no path qualified to be broadened.

Find the shortest path formula is applied for Figure 2.

$$
\mathrm{f}(\mathrm{n})=\mathrm{g}(\mathrm{n})+\mathrm{h}(\mathrm{n})
$$

where $\mathrm{g}(\mathrm{n})$ is the number of trees along with NFC tag and every tag is marked with tag number. So that in here a tree is consider as a node therefore it is noted that both tag and node number are same and $h(n)$, which indicates the distance among the trees with cost, moreover $\mathrm{f}$ (n) selects the shortest path [16]. For example it is considered that there are 6 tags with arbitrary tag number such as: tag 10, tag 11, tag 12, $\operatorname{tag} 13, \operatorname{tag} 14, \operatorname{tag} 15$ and the distance between all the tags are: from tag 10 to 11 the weight is 5 and from 10 to 12 it's 2 , as same 4 for 10 to 13 . So the shortest path formula can be applied here: 


$$
\begin{aligned}
\mathrm{f}(\mathrm{T} 11) & =\mathrm{g}(\mathrm{n})+\mathrm{h}(\mathrm{n}) \\
& =10+5 \\
& =15 \\
\mathrm{f}(\mathrm{T} 10) & =\mathrm{g}(\mathrm{n})+\mathrm{h}(\mathrm{n}) \\
& =5+2 \\
& =7 \\
\mathrm{f}(\mathrm{T} 13) & =\mathrm{g}(\mathrm{n})+\mathrm{h}(\mathrm{n}) \\
& =8+4 \\
& =12
\end{aligned}
$$

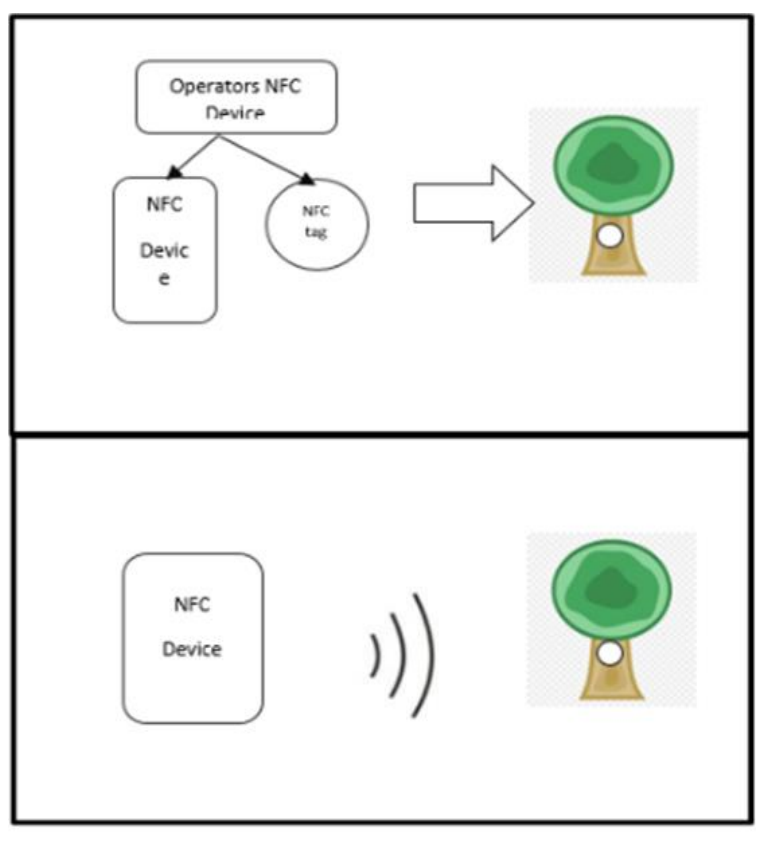

Figure 1. Working procedure

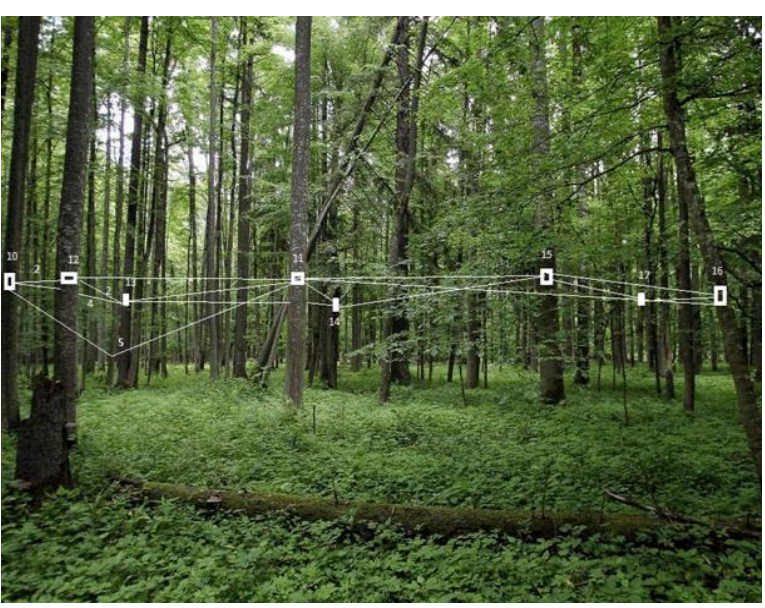

Figure 2. Image of jungle with NFC tag

From the aforementioned calculation it shows that among all the path cost calculation the cost from tag 10 to tag 12 has the lowest path cost. So the optimized next node is tag T12. The searching system will be occurred, stand in front of the NFC tag vertically and has to touch the NFC device to the tag, following information will be showed (i) present tag number (ii) next tag number (iii) distance of desired location (iv) has to move either left, right or any other direction and he will be informed his next instruction through the tag. Figure 3 depicts, applying $A^{*}$ search algorithm formula to find the shortest path in Sundarban forest. It finds the shortest path from the "Start" point to "Goal g". So the shortest path formula is applied here:

$$
\begin{aligned}
& f(a)=20+120=140 \\
& f(d)=10+140=150 \\
& f(b)=32+130=162 \\
& f(d)=10+140=150 \\
& f(b)=32+130=162 \\
& f(C)=25+120=145 \\
& f(b)=32+130=162 \\
& f(h)=65+80=145 \\
& f(b)=32+130=162 \\
& f(g)=75+0=75
\end{aligned}
$$

From the aforementioned calculation it is proved that beginning of the "Start" point to "Goal g" d, e, $\mathrm{h}$ point is the shortest path. 


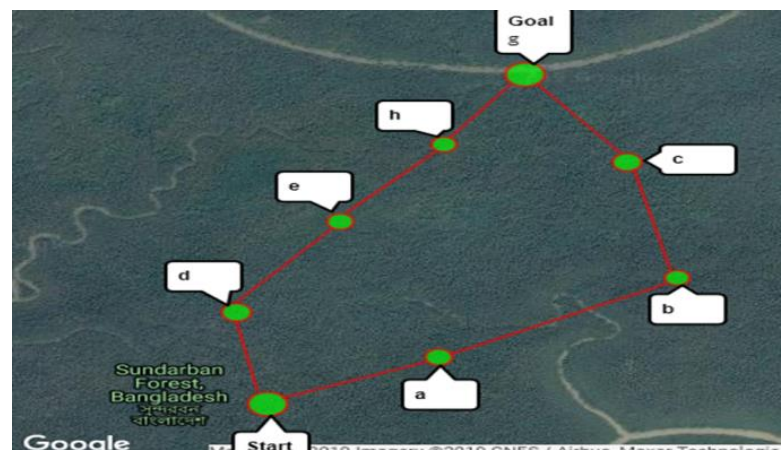

Figure 3. Shortest path on map view

\subsection{A search algorithm}

1 Function reconstruct_path (cameFrom, current)

2 total_path: $=\{$ current $\}$

3 while current in cameFrom.Keys:

4 Current: = cameFrom [current]

5 total_path.prepend (current)

6 return total_path

7 // $\mathrm{A}^{*}$ finds a path from start to goal.

$8 / / \mathrm{h}$ is the heuristic function. $\mathrm{h}(\mathrm{n})$ estimates the cost to reach goal from node $\mathrm{n}$.

9 function A_Star (start, goal, h)

$10 / /$ The set of discovered nodes that need to be (re-)expanded.

$11 / /$ Initially, only the start node is known.

12 openSet $:=\{$ start $\}$

13 //For node $n$, cameFrom[n] is the node immediately preceding it on the cheapest path from start to $n$ currently known.

14 cameFrom: $=$ an empty map

15 //For node n, gScore[n] is the cost of the cheapest path from start to $n$ currently known.

$16 \mathrm{gScore}:=$ map with default value of Infinity

$17 \mathrm{gScore}[\mathrm{start}]:=0$

$18 / /$ For node $n$, fScore[n]:= gScore[n] + h (n).

19 fScore := map with default value of Infinity

$20 \mathrm{fScore}[$ start $]:=\mathrm{h}($ start $)$

21 while openSet is not empty

22 current := the node in openSet having the lowest Score value

23 if current $=$ goal

24 return reconstruct_path(cameFrom, current)

25 openSet.Remove(current)

26 closedSet.Add(current)

27 for each neighbor of current

28 if neighbor in closedSet

29 continue

$30 / / \mathrm{d}$ (current,neighbor) is the weight of the edge from current to neighbor

$31 / /$ tentative_gScore is the distance from start to the neighbor through current

32 tentative_gScore $:=$ gScore[current] + d(current, neighbor)

33 if tentative_gScore $<\mathrm{gScore}[$ neighbor]

34 // This path to neighbor is better than any previous one. Record it!

35 cameFrom[neighbor] := current

$36 \mathrm{gScore}[$ neighbor] $:=$ tentative_gScore

37 fScore[neighbor] := gScore[neighbor] +h(neighbor)

38 if neighbor not in openSet

39 openSet.add(neighbor)

40 // Open set is empty but goal was never reached

41 return failure

42 exit 


\section{RESULTS AND DISCUSSION}

The outcome of this paper is that the percentage of foreign tourists will increase gradually if the proposed method can be physically broadened through the government and they will have a sense of self guide security and become increasingly keen on voyaging. As a result, the economic scenario will be changed. The proposed system is better than the convention human guided system. By this method it is introducing a new potential area of technological era in deep forest. The entire framework works in offline mode it doesn't require any client server communication through internet [17]. So it is a cost-effective system. The full framework can also be implemented by the QR code system [18], but in that case, the user needs to use his or her phone camera to scan the code. Using NFC is more power consuming than the camera [19]. It is mentionable that other wireless technology like Wi-Fi need bandwith for google search to find any path [20]. Travelers can also use hand map for tracking in the forest but there is no technological deployment in this process and this system can be risky for the travelers.

\section{COMPARE WITH THE CONVENTIONAL SYSTEM}

\subsection{Cost effective}

Comparing to the conventional system the proposed study is more cost effective. At present the travelers need to find a tourist guide or a local person and have to make a commercial deal with the person [21]. Besides the proposed framework doesn't require any commercial transection. In Wi-Fi enable network base station or access point functionality is need to provide sevice to the wireless host [22].

\subsection{Independent framework}

The proposed framework is a independent framework comparing to the conventional system. It only requires an one time setup and it doesn't requires any power source to operate the system [23]. Whereas the present situation is fully dependent on the tourist guide as well as the new comer must have to be dependent on the guide. More over any wireless host depend on the base station base backbone [24].

\subsection{Self rescue}

The study framework can make a tourist self-dependent and he or she can execute themselves if they are lost in the deep forest. Besides in the conventional system if a tourist is lost in the jungle he or she must have to wait for his/her guide to rescue him. So the current method is more effective than the conventional system. Application of redio frequency and sensing technology is using to promote the system [25]. More importantly, there is no pre-resistration is required to establish secure communication line like payment transection [26].

\section{CONCLUSION}

In this study, it can be concluded that the design system can be implemented and can make an easy and safe way for the travelers in the deep forest or any other tourist place. The use of NFC makes the transaction be done in a shorter time compared to the use of the conventional system. The utilization of NFC causes the exchange to be done in a shorter time contrasted with the utilization of the conventional framework. For path optimization, the mobile reader traverses from one node to the next, moving around encountered node in its path. The tag perusing procedure is iterative, in which the reader arrives at its start point at the end of each round. Based on the shortest path algorithm that computes the location of tag in the search area is used. NFC tag should be placed so that it will not be separated from the desired tree. Besides, the traveler must have a NFC enabled phone.

\section{REFERENCES}

[1] A. Lazaro, et al., "A survey of NFC sensors based on energy harvesting for IoT applications," Sensors, vol. 18, no. 11, p. 3746, Nov 2018.

[2] S. Nashwan, et al., "Secure Authentication Protocol for NFC Mobile Payment Systems," International Journal of Computer Science and Network Security, vol. 17, no. 8, pp. 256-262, 2017.

[3] G. W. Tan, et al., "NFC mobile credit card: the next frontier of mobile payment?" Telematics and Informatics, vol. 31, no. 2, pp. 292-307, May 2014.

[4] M. Pasquet, et al., "Secure payment with NFC mobile phone in the SmartTouch project," International Symposium on Collaborative Technologies and Systems, pp. 121-126, 2008.

[5] M. Bagus, et al., "Baggage Claim in Airports Using Near Field Communication," Indonesian Journal of Electrical Engineering and Computer Science, vol. 7, no. 2, pp. 442-448, 2017. 
[6] B. Lalithadevi, et al., "Efficient Scheme for Car Parking Using RFID \&NFC," Journal of Network Communications and Emerging Technologies (JNCET), vol. 8, no. 4, pp. 87-92, 2018.

[7] J. Cairó, et al., "NFC system optimization for simultaneous powering and communication with wireless sensors," 2019 European Microwave Conference in Central Europe (EuMCE), Prague, Czech Republic, pp. 517-520, 2019.

[8] M. A. A. Khan, et al., "A Detailed Exploration of Usability Statistics and Application Rating Based on Wireless Protocols," Journal of Advances in Computer Engineering and Technology, vol. 6, no. 1, pp. 9-18, 2020.

[9] K. Vora, et al., "Review on RFID based Audio Tour Guide with NFC Monitoring Function," International Research Journal of Engineering and Technology, vol. 6, no.11, pp. 1261-1264, Nov 2019.

[10] E. Husni, et al., "Mobile Payment Protocol tag-to-tag Near Field Communication (NFC)," International Journal of Interactive Mobile Technologies, vol. 6, no. 4, pp. 34-38, 2012.

[11] E. Husni, et al., "Efficient tag-to-tag Near Field Communication (NFC) Protocol for Secure Mobile Payment," International Conference on Instrumentation, Communication, Information Technology and Biomedical Engineering, pp. 97-101, 2011.

[12] B. Noor, et al., "Near Field Communication (NFC) Protool Using Tag for Secure Mobile Payment," Seminar on Intelligent Technology and Its Application (SITIA), 2012.

[13] N. Sun-Kuk, et al., "Proposal of Micropayment and Credit Card Model using NFC Technology in Mobile Environments," International Journal of Multimedia and Ubiquitous Engineering, vol. 8, no. 3, p. 295, 2013.

[14] M. Nosrati, et al., "Investigation of the*(star) search algorithms: Characteristics, methods and approaches," World Applied Programming, vol. 2, no. 4, pp. 251-256, 2012.

[15] M. Z. Zakaria, et al., "Solving RFID mobile reader path problem with optimization algorithms," Indonesian Journal of Electrical Engineering and Computer Science, vol. 13, no. 3, pp. 1110-1116, Mar 2019.

[16] L. Cheng, et al., "Improved hierarchical A-star algorithm for optimal parking path planning of the large parking lot," International Conference on Information and Automation (ICIA), pp. 695-698, 2014.

[17] J. M. S. Alve, et al., "Smart Outlet: Smart Electrical Outlet with Device Identification Using NFC," Journal of Autonomous Intelligence, vol. 2, no. 4, pp. 21-27, 2019.

[18] Y. Liu, et al., "Recognition of QR Code with mobile phones," Chinese control and decision conference, pp. 203-206, 2008.

[19] M. A. A. Khan, et al., "Machine Learning-Based Driving License Management through Wireless Ad-Hoc Networks Using NFC," International Journal of Recent Technology and Engineering, vol. 8, no. 5, pp. 5146-5152, Jan 2020.

[20] G. Z. Islam, et al., "Achieving Robust Global Bandwidth along with Bypassing Geo-restriction for Internet Users," Indonesian Journal of Electrical Engineering and Computer Science, vol. 18, no. 1, pp. 112-123, Apr 2020.

[21] A. K. Patwary, et al., "Process of Developing a Community Based Tourism and Identifying its Economic and Social Impacts: An Empirical Study on Cox's Bazar, Bangladesh," Pakistan Journal of Humanities and Social Sciences, vol. 7, no. 1, pp. 1-13, 2019.

[22] M. A. A. Khan, et al., "A Study on Delay, Throughput and Traffic Measurement for Wi-Fi Connected Stations Based on MAC Sublayer," American Journal of Computer Science and Engineering Survey, vol. 4, no. 2, pp. 019-028, 2016.

[23] H. Cho, et al., "A Batteryless Chronic Wound Monitoring System with NFC," 2019 IEEE Eurasia Conference on Biomedical Engineering, Healthcare and Sustainability (ECBIOS), Okinawa, Japan, pp. 31-34, 2019.

[24] R. Anand and J. Mungara, "Wireless Environment Aware Adaptive Scheduling Technique for Cellular Networks," Indonesian Journal of Electrical Engineering and Computer Science, vol. 11, no. 1, pp. 318-332, 2018.

[25] L. Cui, et al., "Radio Frequency Identification and Sensing Techniques and Their Applications-A Review of the State-of-the-Art," Sensors, vol. 19, no. 18, p. 4012, 2019.

[26] M. Al-Fayoumi and S. Nashwan, "Performance Analysis of SAP-NFC Protocol," International Journal of Communication Networks and Information Security, vol. 10, no. 1, pp. 125-130, Apr 2018. 